\section{§5. Thermal Transport Due to Turbulence Including Magnetic Fluctuation in Externally Heated Plasma}

Ishizawa, A., Nakajima, N.

We have carried out three-dimensional simulation of a reduced set of two-fluid equations and examined anomalous transport due to electromagnetic microturbulence in externally heated plasma. We applied external heating to turbulent state and examined two cases: one is the thermal transport controlled by external heating, the other is the transport due to coexistence of microturbulence with tearing mode ${ }^{1)}$.

In the case 1 we have investigated temperature profile controlled by external heating. We start numerical simulation from an equilibrium which is unstable against kinetic ballooning mode (micro-instability) and fix the temperature profile. We obtain the turbulent state which is consistent with the fixed temperature profile, then we do not fix the temperature profile and add an external heat source and sink to the turbulent state. The temperature profile does not change between the radius of heat source and heat sink when we add the weak heating and the middle heating that is two times stronger than the weak heating as shown in Fig. 1. This implies profile stiffness. On the other hand we have steeper temperature profile when we add the strong heating that is four times stronger than the weak heating. The transition to the steeper temperature profile occurs about 50 times ion thermal transit time and it is very fast. After adding the external heating, the thermal diffusion for medium heating is about two times larger than that of weak heating. This is consistent with profile stiffness of temperature. On the other hand, the thermal diffusion for strong heating is only three times larger than that of weak heating, in spite of the fact that the strong heating is four times stronger than the weak heating. The zonal flow profile is changed after applying external heating, while the amplification of flow shear is not clear.

In the case 2 we have investigated effects of mutual interactions between micro-turbulence and tearing mode on thermal transport. The initial equilibrium is unstable against kinetic ballooning modes and tearing mode (macro-MHD instability), and the growth rate of former is larger than the latter. The kinetic ballooning modes grow at first, and then a quasi-equilibrium including turbulence is formed for the fixed temperature profile, and it excites $(\mathrm{m}, \mathrm{n})=(2,1)$ mode through nonlinear mode coupling. We remark that this excitation of $(m, n)=(2,1)$ mode by the turbulence is different from destabilization of tearing mode ${ }^{2)}$. After we obtain the quasi-equilibrium including turbulence we do not fix the temperature profile and add heat source and sink that compensates the turbulent heat transport. The quasi-equilibrium including turbulence remains for a while after we add the heat source and sink, then the $(m, n)=(2,1)$ mode becomes similar to tearing mode and grows up. Notice that this mode has different features from standard tearing modes such as large growth rate. These differences will be reported else where. The tearing mode violates magnetic surfaces and produces large-scale magnetic islands, and it affects the micro-turbulence. The magnetic islands reduce the amplitude of dominant Fourier mode of micro-turbulence and increase the energy of low toroidal mode number, and thus the energy spectrum of turbulence is changed. The reduction of micro-turbulence is due to not only the flattening of temperature profile around magnetic islands but also the violation of magnetic surfaces because we have the reduction of micro-turbulence even if we fix the temperature profile ${ }^{2)}$. After the appearance of tearing mode the gradient of ion temperature profile is reduced and both the heat flux and the thermal diffusion coefficient increase around the magnetic islands due to the tearing mode as shown in Fig. 2. We think that the macro-scale flow of tearing mode cooperates with small scale turbulent flow to mix the plasma and produces large thermal diffusion. The zonal flow profile is altered by the appearance of tearing mode $^{2)}$. It seems that the flow shear becomes strong around the magnetic island due to tearing mode (Fig. 2).

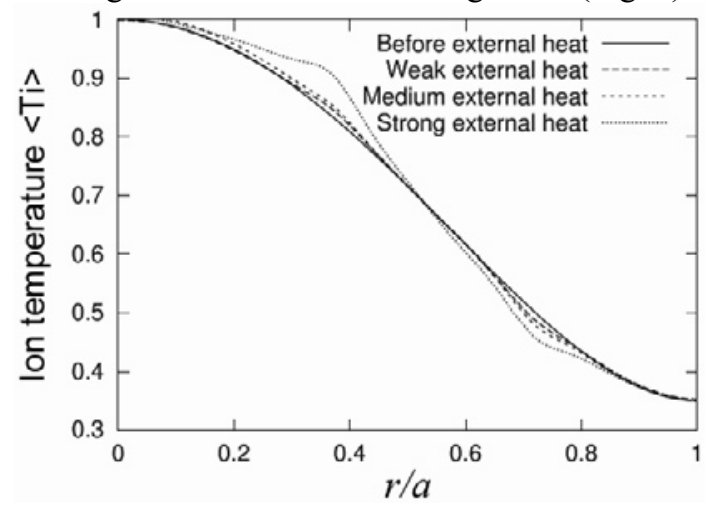

Fig. 1. Ion temperature profile averaged over poloidal and toroidal angles before the additional heating and after in case 1 . The profile does not change around $\mathrm{r} / \mathrm{a}=0.5$ for the weak heating and the medium hearing, while it changes for the strong heating.

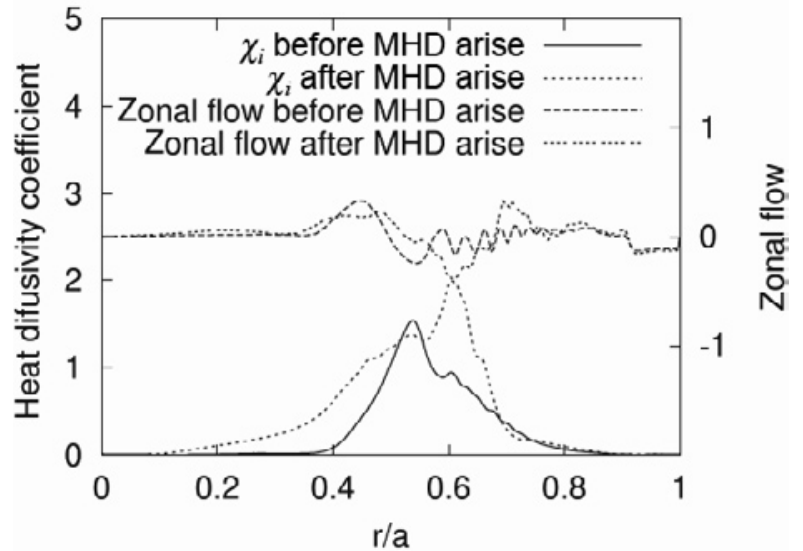

Fig. 2. Radial profiles of thermal diffusion coefficient and zonal flow in case 2 . The thermal diffusivity increases around the magnetic islands due to the tering mode.

1) Ishizawa, A., Nakajima, N., Nuclear Fusion 49 (2009) 055015

2) Ishizawa, A., Nakajima, N., Phys. Plasmas, 14, (2007) 040702 